\title{
Percutaneous Transhepatic Biliary Drainage in Patients with Postsurgical Bile Leakage and Nondilated Intrahepatic Bile Ducts
}

\author{
E.A. de Jong ${ }^{a} \quad$ A. Moelker ${ }^{b} \quad$ T. Leertouwer ${ }^{b} \quad$ S. Spronk ${ }^{c} \quad$ M. Van Dijk ${ }^{d}$ \\ C.H.J. van Eijck ${ }^{a}$
}

Departments of a Surgery, ${ }^{\mathrm{b}}$ Radiology, and ${ }^{\mathrm{c}}$ Epidemiology and Radiology, Erasmus University Medical Centre, and

dDepartments of Pediatric Surgery and Pediatrics, Erasmus MC-Sophia Children's Hospital, Rotterdam, The Netherlands

\section{Key Words}

Percutaneous transhepatic biliary drainage $\cdot$ Bile leakage .

Nondilated bile ducts

\begin{abstract}
Objective and Background: Bile leakage is a serious postoperative complication and percutaneous transhepatic biliary drainage (PTBD) may be an option when endoscopic treatment is not feasible. In this retrospective study, we established technical and clinical success rates as well as the complication rates of PTBD in a large group of patients with postoperative bile leakage. Methods: Data on all patients with nondilated intrahepatic bile ducts who underwent a PTBD procedure for the treatment of bile leakage between January 2000 and August 2012 were retrospectively assessed. Data included type of surgery, site of bile leak, previous attempts of bile leak repair, interval between surgery and PTBD placement. Outcome measures were the technical and clinical success rates, the procedure-related complications, and mortality rate. Results: A total of 63 patients were identified; PTBD placement was technically successful in 90.5\% (57/63) after one to three attempts. The clinical success rate was $69.8 \%$ (44/63). Four major complications were documented (4/63; 6.3\%): liver laceration, pneumothorax, pleural empyema, and prolonged hemobilia. One minor complication in-
\end{abstract}

\section{KARGER}

E-Mail karger@karger.com

www.karger.com/dsu volved pain. Conclusions: PTBD is an effective treatment with low complication rates for the management of postsurgical bile leaks in patients with nondilated bile ducts.

(c) 2014 S. Karger AG, Basel

\section{Introduction}

Postoperative bile leakage is a rare but serious complication of several surgical procedures, such as cholecystectomy, pancreatoduodenectomy, liver resection, and liver transplantation. The incidence ranges from 0.9 to $9.0 \%$ depending on the type of surgical procedure [1-7]. It is associated with significant mortality rates $(8.7-39.0 \%)$ and morbidity $(22-44 \%)[1,3,8,9]$.

Endoscopic and percutaneous transhepatic approaches are both described as effective to resolve biliary obstruction [10-13]. The endoscopic approach via endoscopic retrograde cholangiopancreatography (ERCP) is the first choice $[6,13-16]$. When ERCP is not successful or not feasible, percutaneous transhepatic biliary drainage (PTBD) becomes an alternative. PTBD procedures are most often performed in patients with biliary obstruction and dilated bile ducts, and therefore biliary access can easily be achieved. However, they are also performed in patients with bile leakage and nondilated bile ducts. 
But when the bile ducts are decompressed, many passes are often required to gain access [17].

The first clinical studies on accessing nondilated bile ducts for percutaneous transhepatic cholangiography (PTC) reported a $25 \%$ technical success rate and a $21 \%$ complication rate $[15,18]$. More recent small series have shown that PTC and subsequent drainage for a variety of indications can be feasible in patients with nondilated bile ducts as well; the technical success rates were similar to those in patients with dilated bile ducts (91-100\%) [17, 19-26]. Several studies have described the management of biliary leakage both after hepaticojejunostomy or post-hepatectomy, however a PTBD was performed in the minority of patients $[27,28]$. To our knowledge, we report the largest study on only PTBD procedures for biliary leakage in a group of exclusively postoperative patients. The aim of this retrospective study was primarily to investigate the technical success and also determine the clinical success and complication rates of this procedure.

\section{Materials and Methods}

\section{Patients}

Eligible patients for this study were all subjects who underwent a PTBD procedure for the treatment of postsurgical bile leakage at the Department of Surgery of the Erasmus University Medical Centre, Rotterdam, the Netherlands, between January 2000 and August 2012. Only those patients with nondilated intrahepatic bile ducts on preinterventional ultrasound were included. Patients were subjected to PTBD when there was evidence of persisting bile leakage that could not be treated conservatively or with ERCP. As this study was retrospectively performed, the local ethics committee waived written informed consent.

\section{Variables and Definitions}

We retrieved the following information from the clinical records of the patients included: date of the PTBD procedure, time interval between the initial surgical procedure and the PTBD procedure, type of surgery, site of bile leak, previous attempts of bile leak repair, technical and clinical success rates, procedure-related complications, and patient's survival.

On the preinterventional ultrasound, nondilated ducts were defined as peripheral bile ducts measuring $<2 \mathrm{~mm}$ in diameter or by visualization of ducts smaller than the adjacent portal vein [17].

The initial diagnosis of bile leakage was based on either the presence of bile in a surgical drain or in a percutaneous drain which was placed in a fluid collection on imaging studies performed on clinical findings (e.g. fever, abdominal pain, peritonitis and sepsis). The diagnosis was mostly confirmed by PTC, occasionally by ERCP.

The PTBD procedure was considered to be technically successful if either an internal-external drainage or external drainage only was accomplished. The bile leak was considered clinically successfully resolved if repeat cholangiograms, performed after bile drain production had stopped, showed absence of contrast extravasation outside the bile ducts (definitive closure of the leak). Complications were classified as major or minor according to the Society of Cardiovascular Interventional Radiology guidelines [29]. Major complications are those resulting in hospitalization (for outpatients), unplanned increase in the level of care, prolonged hospitalization, permanent adverse sequelae, or death. Minor complications are those requiring nominal therapy, or a short hospital stay for observation as needed, exclusive of sequelae.

\section{Technique of Percutaneous Biliary Drainage}

Preprocedural antibiotic prophylaxis consisted of $1 \mathrm{~g}$ of Augmentin ${ }^{\circledR}$ administered intravenously. All PTBD procedures were performed under local anesthesia (except when patients were already anesthetized) and patients received intravenous medication for conscious sedation. Under the supervision of an interventional radiologist, fractionated intravenous injection was titrated with steps of 0.05 and $2.5 \mathrm{mg}$ for fentanyl and midazolam, respectively, until effective analgesia and sedation were obtained.

Oxygen saturation and heart rate were monitored during the course of the procedure. The puncture site was chosen under ultrasound guidance and the operation field was disinfected (chlorhexidine, $70 \%$ alcohol) and anaesthetized ( $10 \mathrm{ml}$ of $2 \%$ lidocaine). Next, percutaneous puncture of the intrahepatic bile duct was performed using of a 21-gauge needle (Chiba Tip Needle; Cook Medical, Inc., Bloomington, Ind., USA) under ultrasound guidance. After puncturing the bile duct, contrast material was injected and the correct position of the needle was indicated by opacification of the bile duct. In some cases, if the bile ducts were not visible with ultrasound, the bile duct was punctured under fluoroscopic guidance. The needle was then advanced towards a peripheral portal vein and after removing the needle stylet, contrast was gently injected while withdrawing the needle until a bile duct opacified. When the contrast injection showed that the needle tip was successfully placed in a bile duct, a 0.018 -in guidewire was advanced. The needle was subsequently exchanged for a small coaxial catheter and a cholangiography was made. Next, the small coaxial catheter was replaced by a sheath with a distal marker and a 4-Fr inner diameter using the 0.018 -in guidewire. A catheter with hydrophilic guidewire was then introduced through the 4-Fr sheath and attempts were made to enter the small bowel. Finally, the sheath and catheter were exchanged for a 10-Fr biliary multi-sidehole drainage catheter (Cook Medical, Inc.) over a stiff guidewire for internal-external drainage. If the common bile duct could not be cannulated and no drainage tube could be placed in the small bowel, an 8.5-Fr external drainage catheter was positioned in the common bile duct. After positioning of the PTBD, healing of the bile leak was monitored with repeat cholangiograms.

\section{Data Analysis}

Nominal data are presented as numbers and percentages. Normally distributed variables are presented as mean (SD). Non-normally distributed variables are presented as median (interquartile range). The Mann-Whitney U test was used to compare time between surgery and PTBD for successful procedures versus failed procedures. Fisher exact tests were applied to compare nominal outcomes between groups. 


\section{Results}

More than 900 patients underwent a PTBD procedure for various indications between 2000 and 2012, of whom 63 patients with nondilated bile ducts. The number of PTBD procedures annually varied from 2 to 10 with a median of 4 . The background characteristics of the patients are shown in table 1 . The most frequent bile leakage was seen at the hepaticojejunostomy after liver transplantation or pancreaticoduodenectomy.

The PTBD was performed a median of 17 days after the initial surgical intervention, range 2-664 days (1 patient was treated conservatively for almost 2 years after the initial surgery with multiple ERCP procedures, before undergoing a PTBD procedure). Only 6 patients had a PTBD procedure within 1 week after surgery. In 43 patients (68.3\%) an ERCP was not possible because of postsurgical inaccessibility of the bile ducts. In the remaining 20 patients, PTBD was performed after reported failure of ERCP.

Of the 63 patients, 27 (42.9\%) had undergone a surgical re-exploration before the PTBD. In all these patients a PTBD placement was indicated because the leak could not be found or because the re-exploration did not heal the leak. Of the 63 patients, 8 (12.7\%) underwent the procedure fully anesthetized.

\section{Technical Success}

The overall technical success rate was 90.5\% (57/63). In 51 patients $(81.0 \%)$ the first attempt was successful. In 6 of 12 patients $(50.0 \%)$ in whom the first attempt failed, a second or third attempt at a later stage was successful (fig. 1). In table 2 the technical success rate is shown for the three different sites of bile leakage.

The main outcome measures of the PTBD procedure analyzed per type of surgery are shown in table 3 . The catheter was placed at the right site in 44 patients, at the left site in 11 patients, at both the right and left site in 1 patient, and in 1 patient a right-sided catheter was placed when it failed at the left site.

41 patients received an internal-external drainage catheter, 16 patients an external one. In 5 cases the external drainage catheter was later converted to an internal-external drainage catheter, a mean 12 days (range 7-18) after the initial procedure. In 2 of the 6 patients in whom the PTBD failed, the second attempt failed as well. One of these 2 patients was re-operated and recovered uneventfully. The other was treated with an ERCP and subsequent stent placement, after which the bile leak healed.

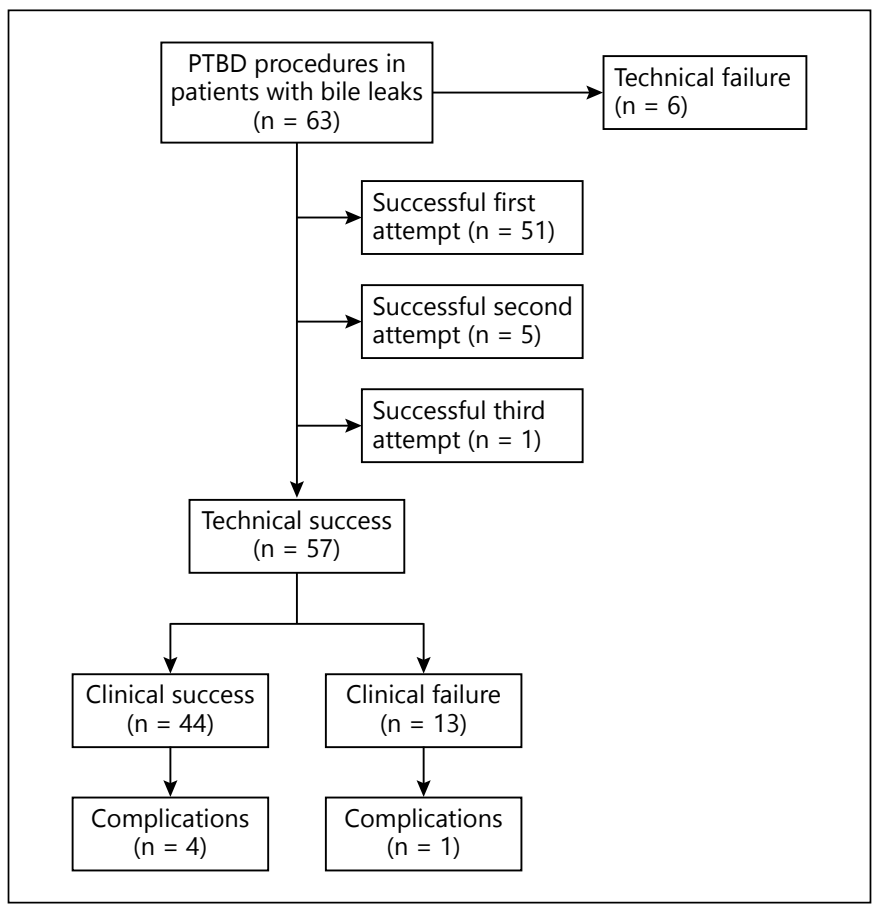

Fig. 1. Flow diagram showing the attempts, successes and failures of the PTBD procedures.

Table 1. Patient characteristics $(n=63)$

\begin{tabular}{ll}
\hline Characteristic & $\mathrm{n}(\%)$ \\
\hline Age, years, mean (SD) & $57(15)$ \\
$\quad$ Range & $21-87$ \\
Male & $31(49)$ \\
Initial surgical intervention & \\
$\quad$ Cholecystectomy & $22(35)$ \\
$\quad$ Biliodigestive anastomosis & $19(30)$ \\
$\quad$ Liver transplantation & $12(19)$ \\
$\quad$ Liver resection & $10(16)$ \\
Site of bile leak & $29(46)$ \\
$\quad$ Bile ducts & \\
$\quad$ Hepatico- or choledochojejunostomy & $30(48)$ \\
Plane of resection & $4(6)$ \\
\hline
\end{tabular}

${ }^{1}$ Leaks at the bile ducts were at the cystic duct stump $(n=4)$, ductus choledochus $(\mathrm{n}=14)$, and common left or right hepatic $\operatorname{duct}(\mathrm{n}=11)$.

Technical success or failure was not statistically significantly related to time between surgery and first PTBD procedure (Mann-Whitney test, $\mathrm{p}=0.13$ ). The technical success rate increased from $82.1 \%$ in the first study period (2000-2006) to $97.1 \%$ in the last study period (20072012) (Fisher exact test, $\mathrm{p}=0.08$ ). 
Fig. 2. A case where the bile leak resolved after the PTBD procedure was successful at the first attempt.

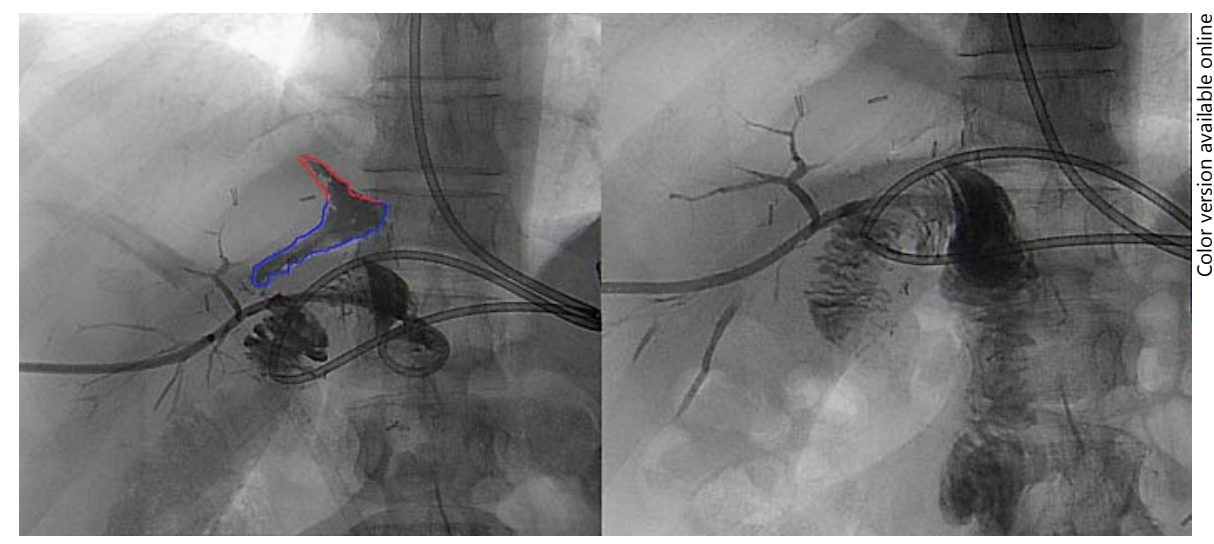

Table 2. Outcome measures by the site of bile leakage, $\mathrm{n}(\%)$

\begin{tabular}{lllll}
\hline Outcome $^{1}$ & $\begin{array}{l}\text { Total sample } \\
(\mathrm{n}=63)\end{array}$ & $\begin{array}{l}\text { Leakage at the bile } \\
\text { ducts }(\mathrm{n}=29)\end{array}$ & $\begin{array}{l}\text { Leakage at the hepatico- or } \\
\text { choledochojejunostomy }(\mathrm{n}=30)\end{array}$ & $\begin{array}{l}\text { Leakage at plane of } \\
\text { resection }(\mathrm{n}=4)\end{array}$ \\
\hline Technically successful & $57(91)$ & $25(86)$ & $28(93)$ & $4(100)$ \\
Clinically successful & $44(70)$ & $19(66)$ & $24(80)$ & $1(25)$ \\
Total complications & $5(8)$ & $1(3)$ & $2(10)$ & $1(25)$ \\
Major complications & $4(6)$ & $1(3)$ & $2(7)$ & $1(25)$ \\
30-Day mortality & $4(6)$ & $1(3)$ & $(25)$ & \\
\hline
\end{tabular}

${ }^{1}$ All Fisher exact tests comparing the four groups on the outcomes of table 2 give a p value of 1.0.

Table 3. Outcome measures by type of surgery

\begin{tabular}{llllll}
\hline Outcome $^{1}$ & $\begin{array}{l}\text { Total sample } \\
(\mathrm{n}=63)\end{array}$ & $\begin{array}{l}\text { Cholecystectomy } \\
(\mathrm{n}=22)\end{array}$ & $\begin{array}{l}\text { Biliodigestive anastomosis } \\
(\mathrm{n}=19)\end{array}$ & $\begin{array}{l}\text { Liver transplantation } \\
(\mathrm{n}=12)\end{array}$ & $\begin{array}{l}\text { Liver resection } \\
(\mathrm{n}=10)\end{array}$ \\
\hline Technically successful & $57(91)$ & $21(95)$ & $15(79)$ & $11(92)$ & $6(50)$ \\
Clinically successful & $44(70)$ & $17(77)$ & $13(68)$ & - & $8(80)$ \\
Total complications & $5(8)$ & $1(5)$ & $3(16)$ & - & $1(10)$ \\
Major complications & $4(6)$ & $1(5)$ & $2(11)$ & $2(17)$ & $1(10)$ \\
30-Day mortality & $4(6)$ & - & $1(5)$ & $10)$ & $1(10)$ \\
\hline
\end{tabular}

${ }^{1}$ All Fisher exact tests comparing the four groups on the outcomes of table 2 give a p value of 1.0.

\section{Clinical Success}

The overall clinical success rate was $69.8 \%$, as the bile leak was completely resolved in 44 of the 63 patients. After a technically successful PTBD, the bile leakage healed in 44 of the $57(77.2 \%)$ patients. In 40 of the 51 patients (78.4\%) in whom the PTBD procedure was successful at the first attempt, the bile leak resolved (an example is shown in figure 2).

Bile leakage at the biliodigestive anastomosis was resolved in $80.0 \%(24 / 30)$ after PTBD placement, leakage at the bile ducts in $65.5 \%(19 / 29)$, and leakage at the plane of resection in $25.0 \%(1 / 4)$ (table 2). However, in 13 patients the bile leak did not resolve; 8 of these patients died before healing of the bile leak could be demonstrated. In the 4 other patients the bile leak was resolved after (a) a new, successful, ERCP procedure with subsequent stent placement $(\mathrm{n}=2),(\mathrm{b})$ an additional left-sided drainage catheter to heal the leak, next to the right-sided catheter that was already present, and (c) by using coils. 
Nine patients with iatrogenic bile duct injury required definitive surgical reconstruction on the biliary tract. They all had clinically successfully resolved bile leaks after PTBD placement. Hepaticojejunostomy was performed in all 9 patients a mean 106 days after PTBD (range 43156). Time between surgery and first PTBD was not statistically significantly different between clinical successes and clinical failures with medians (IQR) of 18 (11-27) and 14 (11-24), respectively (Mann-Whitney $U$ test, $\mathrm{p}=$ $0.52)$. The clinical success rate increased statistically significantly from $60 \%$ in the first study period (2000-2006) to $84.8 \%$ in the second study period (2007-2012) (Fisher exact test, $\mathrm{p}=0.04$ ).

\section{Complications}

The overall complication rate was $7.9 \%$ (5/63). Major complications (6.3\%) documented were: pneumothorax $(n=1)$, pleural empyema $(n=1)$, liver laceration $(n=1)$, and prolonged hemobilia $(\mathrm{n}=1)$. Only one minor complication $(1.6 \%)$ was documented, namely pain after the procedure.

The case of prolonged hemobilia concerned a 75-yearold man with bile leakage after a complicated cholecystectomy after an uncomplicated PTBD procedure. The hemobilia was based on a fistula between the right hepatic artery and the common bile duct. Embolizations and covered stent placements in the hepatic artery successfully stopped the bleeding. All other complications were treated successfully without any clinical sequelae.

The 30-day mortality rate of all patients was $6.3 \%$ (4/63); death occurred 1, 2, 4, and 28 days, respectively, after the PTBD procedure. In-hospital mortality was $11.1 \%(7 / 63)$. These 7 patients were already in a poor condition before the procedure. Six died of underlying problems, i.e. sepsis $(n=4)$, irresectable cholangiocarcinoma $(\mathrm{n}=1)$, and aspiration pneumonia $(\mathrm{n}=1)$. All of them had a technical successful PTBD placement at the first attempt but all, except the patient that died due to an irresectable cholangiocarcinoma, were clinical failures. In the patients who died from sepsis, the PTBD could not control the source of sepsis. No evidence of bile leak resolution was seen at the time of death in the patient who died from aspiration pneumonia.

One patient died of procedure-related complications. This concerned a 50 -year-old woman with persisting bile leakage at the resection plane following a left hepatectomy. Multiple passes were needed before the bile duct was correctly punctured and an internal-external drainage catheter could be placed. Within $1 \mathrm{~h}$ after placement, a liver laceration led to severe hypovolemic, hemorrhagic shock. She was re-operated and packed but the shock could not be corrected and she died the next day.

\section{Discussion}

This study showed that PTBD is an effective treatment for the management of postsurgical bile leaks, as the bile leak resolved in $69.8 \%(44 / 63)$ of cases. The technical success rate was high at $90.5 \%$ and the complication rate was low at $7.9 \%$. To the best of our knowledge, this study encompassed the largest time span and sample size of its kind. Over the last 20 years a number of small studies in at least 10 patients also reported high technical success rates $(91-100 \%)$, high clinical success rates $(70-100 \%)$, and low major complication rates $(0-13 \%)[8,19,21,22$, $25,26,30]$.

\section{Technical Success}

The above high technical success rates were achieved despite nondilated bile ducts. In the present study we used ultrasound guidance either alone or, when bile ducts were not visible or could not be punctured directly, combined with fluoroscopy. In a study from Kuhn et al. [19] the fluoroscopy time in patients with nondilated bile ducts was significantly longer than that in patients with dilated bile ducts. These authors also applied supplementary techniques for the opacification of the intrahepatic bile system: in 16 of 21 patients they used either CT-guided percutaneous puncture, T-drainage, or a temporary gallbladder drainage to aid the PTBD placement, resulting in a $100 \%$ technical success rate. Aytekin et al. [20] achieved access to dilated bile ducts with percutaneous puncture of a peripheral duct under ultrasonographic guidance. In patients with nondilated peripheral bile ducts at ultrasound, the puncture was performed under fluoroscopic guidance using cholangiography (obtained via the drainage catheter near the leak site or surgical T-tube). Furthermore, in some studies the puncture site was usually more central when the bile ducts were nondilated, as this facilitates the puncture $[21,22,26]$. Also, Funaki et al. [17] reported a higher number of passes in patients with nondilated bile ducts. In the present study, too, the puncture site was occasionally more central, and in some cases multiple passes were needed before the bile ducts were correctly punctured. Since the radiologist performing these procedures remained the same over the study period, the increase of the success rate $(97.1 \%)$ in the last study pe- 
riod (2007-2012) compared to $82.1 \%$ in the first study period (2000-2006) is probably due to the improvement of imaging modalities, especially the ultrasound used for PTBD.

\section{Clinical Success}

The overall clinical success rate in this study was $69.8 \%$ $(44 / 63)$, a little lower than rates reported in other studies, ranging from 70 to $100 \%[8,21,22,25,26,30]$. The difference can be explained by the complexity and the severity of the underlying diseases of our patients. Our hospital serves as a tertiary university-based referral center for liver pathology, and many patients were already in a very poor condition when they arrived, presenting themselves with concomitant abdominal sepsis.

The increased clinical success rate, from $60 \%$ in the first period to $84.4 \%$ in the second study period, was mainly due to the technical progress made by our radiologist, which led to a higher percentage of patients with a successful PTBD procedure.

\section{Early versus Late PTBD}

Statistical analysis showed that the interval between initial surgical procedure and PTBD procedure is not related to the technical and clinical success rates, which is probably due to the heterogeneity of our patient group. However, in patients with signs of sepsis due to the bile leakage, early PTBD is most likely indicated and may be less successful due to the patient's poor clinical condition. Early drainage in itself may have a negative effect on technical and clinical success rates.

\section{Complications}

We found an overall complication rate of 7.9\% (5/63); 4 patients developed major complications (6.3\%), 1 of whom died. Comparisons with other studies are impeded by the different definitions used. However, those studies with a similar major complication definition reported major complication rates from 0 to $13 \%[8,17,19-26,31$, 32 ]. Our major complication rate of $6.3 \%$ fits within this range.

As mentioned above, nondilated bile ducts are sometimes punctured more centrally. However, puncture closer to the hepatic hilum, and therefore also closer to larger vascular structures, carries a higher risk of vascular complications [33]. Only 1 (1.6\%) vascular complication was documented in our study. Similar series report vascular complications after the PTBD procedure in $4-13 \%$ of patients with bile leaks $[8,22,26,30]$. These numbers should be interpreted with caution though, as the patient popula- tions in these studies were relatively small. Still, the major complication rates of most studies are below the $10 \%$ threshold recommended by the Society of Cardiovascular and Interventional Radiology [29].

For the different types of surgery as well as types of bile leak, we found only slight differences in the main outcomes. This, in combination with the small numbers of patients in each group, does not allow making definite clinical recommendations.

Several limitations of the study need to be addressed. Firstly, it is a retrospective study over a 12 -year period with its inherent disadvantages. Information on the number of punctures, procedure time and minor complications was not always available, therefore we only scored for separate radiological attempts. Secondly, PTBD procedures were sometimes performed under ultrasound guidance alone and as ultrasound is an operator-dependent procedure, the results will differ with the experience of the operator. Thirdly, the patient group was heterogeneous with respect to the underlying disease and cause of biliary leakage, but nevertheless represents clinical practice. Fourthly, as some studies report that bile leakage is associated with biliary anastomotic strictures $[8,17,19-$ $26,31,32]$, we attempted to analyze the occurrence of strictures following a PTBD procedure. However, we could not draw conclusions due to the fact that many patients returned to their former hospital for follow-up after treatment. Lastly, because length of hospital stay in other settings was unknown, we could not draw conclusions on duration of treatment.

In conclusion, this study showed that PTBD is an effective treatment with low complication rates for the management of postsurgical bile leakage in patients with nondilated bile ducts.

References
1 Buanes T, et al: Bile leak after cholecystectomy significance and treatment: results from the National Norwegian Cholecystectomy Registry. Int Surg 1996;81:276-279.

$\checkmark 2 \mathrm{Li}$ SQ, et al: Bile leakage after hepatectomy for hepatolithiasis: risk factors and management. Surgery 2007;141:340-345.

-3 Lo CM, et al: Biliary complications after hepatic resection: risk factors, management, and outcome. Arch Surg 1998;133:156-161. cidence of pancreatic and biliary leakages after pancreaticoduodenal resections. Hepatogastroenterology 1996;43:1246-1249.

5 Yeo CJ, et al: Six hundred fifty consecutive pancreaticoduodenectomies in the 1990s: pathology, complications, and outcomes. Ann Surg 1997;226:248-260.
4 Parviainen MC, Sand JA, Nordback IH: Coin- 
-6 Akamatsu N, et al: Biliary reconstruction, its complications and management of biliary complications after adult liver transplantation: a systematic review of the incidence, risk factors and outcome. Transpl Int 2011;24: 379-392.

$7 \mathrm{Kim} \mathrm{JH}$, et al: Management of patients who return to the hospital with a bile leak after laparoscopic cholecystectomy. J Laparoendosc Adv Surg Tech A 2010;20:317-322.

$>8 \mathrm{Kim} \mathrm{JH}$, et al: Bile leak following living donor liver transplantation: clinical efficacy of percutaneous transhepatic treatment. Liver Transpl 2008;14:1142-1149.

$>9$ Yamashita Y, et al: Bile leakage after hepatic resection. Ann Surg 2001;233:45-50.

$>10$ Schumacher B, et al: Long-term follow-up of percutaneous transhepatic therapy in patients with definite benign anastomotic strictures after hepaticojejunostomy. Endoscopy 2001; 33:409-415.

11 Weber A, et al: Long-term outcome of endoscopic and/or percutaneous transhepatic therapy in patients with biliary stricture after orthotopic liver transplantation. J Gastroenterol 2009;44:1195-1202.

$12 \mathrm{Kim} \mathrm{JH}$, et al: Percutaneous transhepatic cholangioscopic treatment of patients with benign bilio-enteric anastomotic strictures. Gastrointest Endosc 2003;58:733-738.

$>13$ Lai EC, et al: Endoscopic biliary drainage for severe acute cholangitis. N Engl J Med 1992; 326:1582-1586.

14 Kumar R, et al: Endoscopic biliary drainage for severe acute cholangitis in biliary obstruction as a result of malignant and benign diseases. J Gastroenterol Hepatol 2004;19:994997.
15 Teplick SK, et al: Transhepatic cholangiography in patients with suspected biliary disease and nondilated intrahepatic bile ducts. Gastrointest Radiol 1991;16:193-197.

16 McLindon JP, et al: Causes, clinical features and non-operative management of bile leaks. Eur Radiol 1998;8:1602-1607.

17 Funaki B, et al: Percutaneous biliary drainage in patients with nondilated intrahepatic bile ducts. AJR Am J Roentgenol 1999;173:15411544.

18 Elias E: Cholangiography in the jaundiced patient. Gut 1976;17:801-811.

19 Kuhn JP, et al: Percutaneous biliary drainage in patients with nondilated intrahepatic bile ducts compared with patients with dilated intrahepatic bile ducts. AJR Am J Roentgenol 2010;195:851-857.

20 Aytekin C, et al: Percutaneous management of anastomotic bile leaks following liver transplantation. Diagn Interv Radiol 2007;13:101104.

21 Cozzi G, et al: Percutaneous transhepatic biliary drainage in the management of postsurgical biliary leaks in patients with nondilated intrahepatic bile ducts. Cardiovasc Intervent Radiol 2006;29:380-388.

22 Ernst O, et al: Biliary leaks: treatment by means of percutaneous transhepatic biliary drainage. Radiology 1999;211:345-348.

23 Lee W, et al: Ultrasound and fluoroscopy guided percutaneous transhepatic biliary drainage in patients with nondilated bile ducts. Abdom Imaging 2008;33:555-559.
24 Pedicini V, et al: Management of post-surgical biliary leakage with percutaneous transhepatic biliary drainage and occlusion balloon in patients without dilatation of the biliary tree: preliminary results. Eur Radiol 2010;20: 1061-1068.

25 Righi D, et al: Safety and efficacy of the percutaneous treatment of bile leaks in hepaticojejunostomy or split-liver transplantation without dilatation of the biliary tree. Liver Transpl 2008; 14:611-615.

26 Stampfl U, et al: Percutaneous management of postoperative bile leaks after upper gastrointestinal surgery. Cardiovasc Intervent Radiol 2011;34:808-815.

27 Hoekstra LT, et al: Posthepatectomy bile leakage: how to manage. Dig Surg 2012;29:48-53.

28 De Castro SM, et al: Incidence and management of biliary leakage after hepaticojejunostomy. J Gastrointest Surg 2005;9:1163-1173.

29 Burke DR, et al: Quality improvement guidelines for percutaneous transhepatic cholangiography and biliary drainage. J Vasc Interv Radiol 2003;14:S243-S246.

30 Aytekin C, et al: Percutaneous therapy for anastomotic bile leak in liver-transplant patients with nondilated bile ducts. Cardiovasc Intervent Radiol 2007;30:761-764.

-31 Kaufman SL, et al: Percutaneous transhepatic biliary drainage for bile leaks and fistulas. AJR Am J Roentgenol 1985;144:1055-1058.

32 Weber A, et al: Complications of percutaneous transhepatic biliary drainage in patients with dilated and nondilated intrahepatic bile ducts. Eur J Radiol 2009;72:412-417.

-33 L'Hermine C, et al: Arterial complications of percutaneous transhepatic biliary drainage. Cardiovasc Intervent Radiol 1996;19:160-164. 